\title{
ANALYSIS OF 30 WOLF-RAYET STARS
}

\author{
W. Schmutz, W.-R. Hamann, U. Wessolowski \\ Institut für Theoretische Physik und Sternwarte der Universität Kiel \\ 01 shausenstrasse, D-2300 Kiel 1, Federa? Republic of Germany
}

Temperatures, mass-loss rates and luminosities of 30 galactic Wolf-Rayet stars (24 $W N, 6 W C$ ) are derived by fitting the observed equivalent widths of He I $\lambda 5876$ and He II $\lambda 5412$ and the absolute visual magnitude. A three-dimensional grid $\left(T_{\star}-R_{\star}-\dot{M}\right)$ of model calculations provides the theoretical values.

The results are summarized in Figure 1. The different symbols denote the spectral classification of the individual stars, whereas four groups are distinguished: WNE$A$, WNE-B, WNL and WC stars. The size of the symbols indicates the mass-loss rates. The uncertainties of the results are estimated to be 0.1 dex in $T_{\star}, 0.4$ dex in $\dot{M}$ and 0.5 dex in L. For the 11 stars in common with the sample of Abbott et al. (1986) we find our mass-loss rates to be compatible with their radio flux if the correct ionization equilibrium in the radio emitting region is applied (Schmutz and Hamann, 1986). The model calculations show that for all but the WN2 and WN3 stars helium recombines to $\mathrm{He}^{+}$before the ions enter the radio-emitting region.

The results for the four groups are discussed briefly in the following:

- WNE-A: subtypes WN2 to WN6 with weak ("A") emission lines

The stars of this group do not form a homogeneous set. Therefore, no typical parameters can be assigned to them, except that they have relatively small massloss rates. Only a lower limit of $70 \mathrm{kK}$ can be set to the temperatures of the WN2 and WN3 stars, because no He I lines are observed in their spectra. The other WNE-A stars have temperatures between 33 and 50kK. For the only binary of our sample, V444 Cyg, two solutions are given in Fig. 1. (brackets) for two different adopted absolute magnitudes, -4.0 or -4.8 , respectively.

- WNE-B: subtypes WN4 to WN6 with strong ("B") emission lines

This group is characterized by high mass-loss rates, and by luminosities of about $10^{5.5} \mathrm{~L}_{\theta}$. Their effective temperatures, referred to the core radius, are between 50kK and 90kK. But, owing to their high mass-loss rates, the continuum of the WNE-B stars emerges from regions in the wind. If the effective temperatures are referred to the radius of optical depth $2 / 3$, they become $40 \mathrm{kK}$ or less for all stars of this group. The difference between core radius and continuum-emitting region is only important for the WNE-B stars. 
- WNL: subtypes WN7 to WN9

All stars of this group have almost the same temperature. Therefore a similar bolometric correction, BC, of about $3 \mathrm{mag}$ applies for these stars. Because the temperatures are close to the threshold where the He II emissions break down, different spectral subtypes appear over a small range of temperatures.

- WC: subtypes WC5 to WC9

The WC stars have lower luminosities than the WN stars. This is a consequence of their relatively low absolute visual magnitudes, together with their comparably low temperatures, which are resulting from the ratio of the strong He I lines (at $10830 \AA$ ) to the rather weak He II emissions at $5412 \AA$. The stellar temperatures of the WC stars range from $40 \mathrm{kK}$ for subtype WC5 to $30 \mathrm{kK}$ for WC9.

It is obvious that the derived parameters are not in agreement with the "standard" evolutionary calculations leading to Wolf-Rayet stars (Maeder and Meynet, 1987). The most extreme disagreement is found for the WC stars.

A detailed version of this paper is in preparation for "Astronomy and Astrophysics".

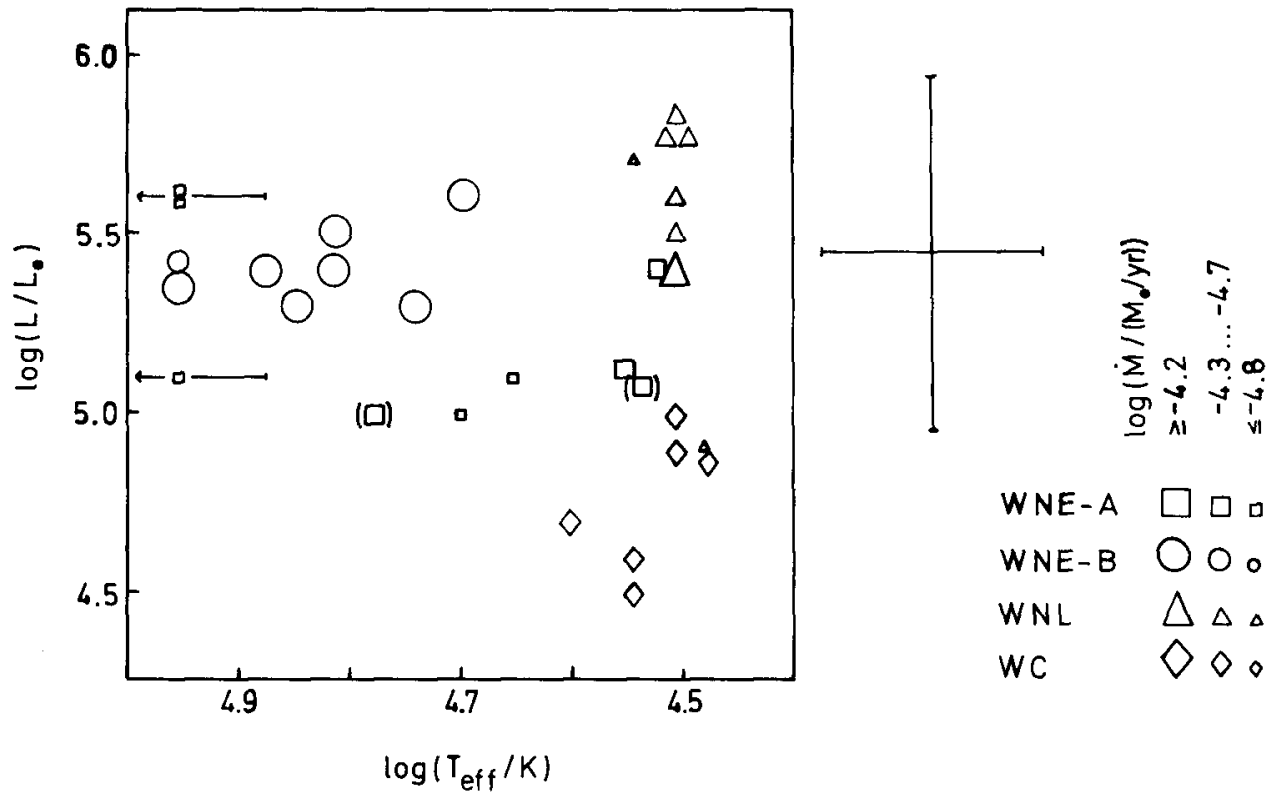

Figure 1. The location in the HR-diagram of the 30 Wolf-Rayet stars as resulting from this work. The effective temperatures are referred to the "core" radii

\section{REFERENCES}

Abbott,D.C., Bieging,J.H., Churchwe11,E., Torres,A.V.: 1986, Astrophys. J. 303, 239 Schmutz,W., Hamann,W.-R.: 1986, Astron. Astrophys. Letter 166, L11

Maeder,A., Meynet,G.: 1987, Astron. Astrophys. 182, 243 